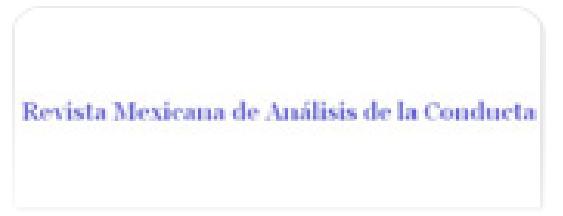

Revista Mexicana de Análisis de la Conducta ISSN: 0185-4534

editora@rmac-mx.org

Sociedad Mexicana de Análisis de la Conducta México

Bachá Méndez, Gustavo; Reid, Alliston K.

Adquisición de patrones simples de respuestas

Revista Mexicana de Análisis de la Conducta, vol. 32, núm. 2, diciembre, 2006, pp. 155-177

Sociedad Mexicana de Análisis de la Conducta

Guadalajara, México

Disponible en: http://www.redalyc.org/articulo.oa?id=59332205

Cómo citar el artículo

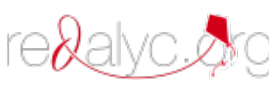

- Número completo

- Más información del artículo

- Página de la revista en redalyc.org

Sistema de Información Científica

Red de Revistas Científicas de América Latina, el Caribe, España y Portugal Proyecto académico sin fines de lucro, desarrollado bajo la iniciativa de acceso abierto 


\title{
ADQUISICIÓN DE PATRONES SIMPLES DE RESPUESTAS
}

\author{
ACQUISITION OF SIMPLE PATTERNS OF RESPONSES \\ GUSTAVO BACHÁ MÉNDEZ1 Y ALLISTON K. REID² \\ ${ }^{1}$ FACULTAD DE PSICOLOGÍA, UNAM \\ ${ }^{2}$ WOFFORD COLLEGE, S C
}

\begin{abstract}
RESUMEN
Para analizar el impacto de cambios frecuentes en las consecuencias sobre la adquisición de patrones simples de conducta, seis ratas recibieron alimento por emitir una de cuatro posibles secuencias de dos respuestas en una caja de condicionamiento operante con dos palancas. Para cuatro animales, el reforzador siguió a una de las secuencias durante los primeros 50 ensayos de la sesión y a una secuencia diferente durante los últimos 50 ensayos. En cada sesión se utilizó una de 12 combinaciones de secuencias posibles y el ciclo de estas 12 sesiones se repitió en seis ocasiones. Lo anterior aseguró que cada secuencia tuviera un número igual de oportunidades de ser reforzada. Para un segundo grupo, se seleccionó de manera aleatoria una secuencia para ser reforzada y esta contingencia se mantuvo hasta que se obtuvo el reforzador, entonces se seleccionó una nueva secuencia. De esta manera cada secuencia recibió un número similar de reforzadores. La distribución de frecuencias de las secuencias fue diferente para los grupos. Al examinar la dinámica de las respuestas ante los cambios de contingencia se detectaron procesos a nivel de las respuestas individuales más que a nivel de unidades
\end{abstract}

1. El trabajo es parte de los requisitos para la obtención del grado de Doctor en Psicología del primer autor. Parte de este estudio se presentó en el XV Congreso Mexicano de Psicología en Guanajuato, Gto. en septiembre de 2004. Agradecemos a Olivia Maciel por la conducción del experimento, a Erika Gutiérrez M., Alejandro Rangel S. por sus comentarios y en especial a Ixel Alonso O. y Diana Herrera E. por su valiosa colaboración en el análisis de los datos y revisión del texto. Dirigir la correspondencia al primer autor al Laboratorio de Adaptación Animal, Facultad de Psicología, UNAM., Cub. 22, Edificio "D”, Ave. Universidad núm. 3004, Colonia Copilco Universidad, CP: 04510. México, D.F. o al correo bacha@servidor.unam.mx 
complejas de conducta. El procedimiento utilizado promete ser una técnica útil en el estudio de la adquisición de patrones conductuales simples.

Palabras clave: patrones conductuales, secuencias de respuestas, adquisición, palancas, ratas.

\section{SUMMARY}

In order to study how simple patterns of responses are influenced by their consequences, six rats received food for producing one of four possible sequences of two responses in an operant conditioning chamber with two levers. For four animals, one sequence was reinforced for the first 50 trials of the session, and a different sequence was reinforced for the last 50 trials. In each session one of 12 possible sequences combinations was used, and this cycle of these 12 sessions was repeated on six occasions. This procedure assured that every sequence had an equal number of opportunities of being reinforced. For a second group of rats the reinforced sequence was selected at random. This contingency was maintained until one reinforcer was delivered, and the next reinforced sequence was again selected at random. Thus, every sequence received similar number of reinforcements each session. The frequency distribution of the sequences was different for the two groups. Examination of the dynamics of responding when contingencies were changed revealed processes that acted on individual responses, rather than processes that acted at the level of sequences as complex behavioral units. This procedure promises to be a useful technique for the study of the acquisition of simple behavioral patterns.

Key words: behavioral patterns, response sequences, acquisition, levers, rats.

Una cadena o secuencia de respuestas es considerada como un conjunto de respuestas que mantiene un orden particular y que de este orden depende la entrega del reforzador (Catania, 1998; Kelleher, 1966; Skinner, 1938). Diferentes autores han ayudado en el descubrimiento de los mecanismos que permiten la formación de tales secuencias conductuales. Por ejemplo, Catania (1971) utilizó programas tandem: intervalo variable (IV) más una secuencia de respuestas, para mostrar que el reforzador no sólo tiene efecto sobre la respuesta a la que sigue, sino sobre todo el patrón de respuestas que anteceden a la última respuesta. Cada respuesta fortalecida colabora entonces de manera independiente a la tasa total de respuesta observada, pero la proporción con la que contribuye depende de su posición en la secuencia, esto es, de su distancia al reforzador. En otro estudio, Grayson y Wasserman 
(1979) revelaron que es posible diferenciar entre varias secuencias al hacer depender el reforzador de sólo una de ellas. La frecuencia de está secuencia que recibía el reforzador fue diferente que aquellas que no eran seguidas de alimento. Estos autores concluyen que el orden mismo de las respuestas es una propiedad discriminable de la conducta. En su discusión consideraron dos tipos de explicación, una que parte del trabajo de Catania (1971), basada en los efectos de contigüidad y otra que sugiere que la unidad funcional incluye la información contenida en el sistema de memoria a corto plazo del animal (Shimp, 1979). Por su parte Fetterman y Stubbs (1982) reforzaron de manera concurrente cuatro diferentes secuencias de dos respuestas usando dos operandos. Estos autores manipularon diferentes combinaciones de probabilidad de reforzamiento para cada secuencia. Sus resultados indican, sorprendentemente, que la igualación se presentó muy bien entre las secuencias, pero no entre respuestas individuales.

En una serie de trabajos más reciente, Reid (1994), y Reid, Dixon y Gray (en prensa) argumentaron y probaron que si el reforzador actúa sobre las respuestas individuales y no sobre el conjunto completo de ellas, entonces el conjunto de respuestas no es una unidad, aun cuando mantenga su estructura ante cambios de contingencia. Estos autores, además, mostraron que la conducta de las ratas se ve influida por la estructura de la secuencia aprendida previamente, así como por la estructura de la nueva secuencia a aprender. En contra de la idea aceptada de que una respuesta con más fuerza debe persistir más ante cambios en la contingencia, Reid y sus colaboradores, demostraron que la fuerza de la respuesta no se expresa como una persistencia ante cambios de contingencia sino como una mayor capacidad o sensibilidad para cambiar. Esto significa que si la nueva secuencia era diferente a la anterior en la respuesta más cercana al reforzador, entonces era más rápido aprender esta nueva secuencia.

En todos estos trabajos, los procedimientos representan un ambiente relativamente constante, porque las condiciones se mantienen por periodos que incluyen varias sesiones y luego de alcanzar un criterio de estabilidad se cambia la secuencia de la que dependerá la entrega del alimento. Por otro lado, se ha utilizado la estrategia de realizar cambios frecuentes en las condiciones de reforzamiento, por ejemplo Polidora (1963) utilizando ratas y Boren y Devine (1968) trabajando con monos rhesus, usaron un procedimiento de adquisición repetida de secuencias, con el propósito de tener una línea base y probar el efecto de diferentes fármacos y niveles de privación. Thompson $(1973,1975)$ usó adquisiciones repetidas de secuencias de cuatro respuestas emitidas en tres operandos, incluyó el control de la posición de cada respuesta en la secuencia, además de hacer cambios en la asignación del reforzador a las diferentes secuencias en cada sesión. Estos trabajos son muy valiosos, pero su propósito fue utilizar la secuencia como una línea base para probar 
el efecto de diferentes fármacos, dejando pendiente el estudio de las condiciones que permiten la formación de las secuencias mismas. No obstante, el procedimiento es interesante porque enfatiza la importancia de mantener el control del número de oportunidades que tiene cada una de las secuencias estudiadas para obtener el reforzador, acción ésta, que facilita la comparación entre diferentes condiciones en las que se forma una secuencia.

Siguiendo esta línea de trabajo y la de autores como Catania (1971), Grayson y Wasserman (1979) y Reid, A. K., Chadwick C. Z., Dunham, M. \& Miller A., (2001) el presente trabajo tiene el propósito de estudiar la formación de patrones simples de conducta como una forma de adaptación conductual a cambios frecuentes en las contingencias de reforzamiento. El uso de secuencias de respuestas permite estudiar el efecto de la estructura de la conducta, al hacer depender la entrega del reforzador del orden en el que se emiten las respuestas individuales. Para un grupo se controla el número de oportunidades de obtener el reforzador con cada secuencia, mientras que para otro grupo se regula el número de reforzadores que se obtendrán por cada secuencia. Así para un grupo las oportunidades son iguales pero la estructura puede hacer la diferencia, mientras que para el otro grupo el número de reforzadores es el mismo y se puede observar el efecto de esto, en secuencias con diferente estructura.

El procedimiento también permitirá observar algunos efectos como los atribuidos a la posición de cada elemento dentro de la secuencia con respecto al reforzador. Así como la influencia de la primera secuencia aprendida en la sesión sobre la segunda, mientras la primera se encuentra en proceso de extinción.

\section{MÉTODO}

\section{Sujetos}

Seis ratas albinas de tres meses de edad al inicio del estudio y sin experiencia previa en procedimientos de condicionamiento operante. Los animales fueron alojados en cajas habitación estándar, con agua siempre disponible y en un régimen de privación de alimento que los mantuvo en el $85 \%$ de su peso ad libitum.

\section{Aparatos}

Dos cajas de condicionamiento operante de la compañía MED colocadas dentro de cajas sono-amortiguadoras de $60 \times 90 \times 80 \mathrm{~cm}$ con un extractor de aire que enmascaró ruidos externos. En la sección central del panel frontal, a $1 \mathrm{~cm}$ del piso, se encontraba una abertura cuadrada de $3 \mathrm{~cm}$ por lado, que 
daba acceso a un receptáculo en el que se entregaban pellas de $45 \mathrm{mg}$ (fórmula $F$ de Noyes). A cada lado de este orificio se encontraban dos palancas, una colocada a la izquierda y que será denominada palanca "I" y otra colocada a la derecha identificada como palanca " $D$ ". Para su funcionamiento cada palanca requería de una fuerza de $0.15 \mathrm{~N}$, y tenían un foco $28 \mathrm{~V} \mathrm{DC}$ colocado a $3 \mathrm{~cm}$ por encima de ellas; un foco similar colocado en la pared posterior cerca del techo sirvió como luz general. La programación y el registro de cada evento junto con su tiempo de ocurrencia se hicieron mediante una computadora personal y una interfase de la misma compañía MED.

\section{Procedimiento}

Entrenamiento. En las tres primeras sesiones los animales trabajaron con un programa que entregaba una pella después de dos respuestas, sin importar si se utilizaba una o las dos palancas. Estas sesiones concluían cuando los sujetos obtenían 50 reforzadores o transcurrían $30 \mathrm{~min}$. Durante las siguientes sesiones, se entregaba el alimento sólo si el sujeto respondía de manera alternada, ejecutando una respuesta en cada palanca, sin importar el orden. Una vez que los sujetos cumplieron con el requisito de obtener 50 reforzadores en tres sesiones consecutivas, fueron asignados de forma aleatoria a uno de dos grupos: cuatro animales a un grupo llamado de cambios intra-sesión (CIS) y dos sujetos más a otro grupo en el que la selección de la secuencia reforzada fue aleatoria (SA).

Procedimiento experimental. En ambos grupos se usaron las secuencias que resultan de la combinación de dos respuestas utilizando las palancas I y D (II, ID, DI y DD). En el grupo CIS, sólo una de las secuencias fue seguida de alimento en cada ensayo. Cada ensayo iniciaba con el encendido de la luz general y de las luces colocadas por encima de cada una de las palancas, y concluía con la segunda respuesta del sujeto. En el caso de que la secuencia emitida fuera correcta, se entregaba una pella al mismo tiempo que se apagaban la luz general y las luces sobre las palancas, por un período de 3 segundos antes de iniciar el siguiente ensayo. Si la secuencia emitida era incorrecta, todas las luces se apagaban durante 10 segundos y no se entregaba alimento. Durante los primeros 50 ensayos de cada sesión se reforzaba una secuencia y a partir del ensayo 51 se reforzó una secuencia diferente, el ensayo número 100 marcaba el final de la sesión. La secuencia que era reforzada del ensayo 51 al 100 era utilizada como la primera secuencia en la siguiente sesión (véase la parte izquierda de la tabla 1). Cada sujeto fue expuesto a un ciclo de 12 sesiones, cada sesión con una de las 12 combinaciones mostradas a la derecha de la tabla 1. Para cada sujeto, estas combinaciones se presentaron en un orden diferente y el ciclo fue repetido seis veces antes de concluir el experimento. 
Tabla 1. Se muestra el orden de reforzamiento para una secuencia en los primeros 50 ensayos y para una diferente durante los últimos 50 ensayos de cada sesión. Del lado derecho se muestran las 12 combinaciones utilizadas en cada ciclo. Este ciclo se repitió seis veces a lo largo del estudio.

\begin{tabular}{|c|c|c|c|c|c|c|}
\hline \multirow[b]{2}{*}{ SESIONES } & \multicolumn{2}{|c|}{ ENSAYOS } & & & & \\
\hline & $1-50$ & $51-100$ & \multirow{2}{*}{\multicolumn{4}{|c|}{ COMBINACIONES }} \\
\hline & & & & & & \\
\hline 1 & ID & II & II---ID & ID---II & DI---II & DD---II \\
\hline 2 & II & DI & II---DI & ID---DI & DI---ID & DD---ID \\
\hline 3 & DI & DD & II---DD & ID---DD & DI---DD & DD---DI \\
\hline . & . & - & & & & \\
\hline$\cdot$ & $\cdot$ & $\cdot$ & & & & \\
\hline$\dot{12}$ & $\dot{\mathrm{DD}}$ & $\dot{\text { ID }}$ & & & & \\
\hline
\end{tabular}

Los sujetos en el grupo SA fueron expuestos durante 30 sesiones a un programa que elegía una de las cuatro secuencias de forma aleatoria (con reemplazo) y con una probabilidad igual para cada una de ellas. El programa comparaba su elección con las respuestas del animal. Si la secuencia emitida por el sujeto era diferente, entonces se iniciaba un periodo de 10 segundos de tiempo fuera como ocurrió en el grupo CIS y se comenzaba otro ensayo manteniendo la misma secuencia meta. En cambio, si las respuestas del sujeto coincidían con la secuencia elegida por el programa, se presentaba el alimento, y se apagaban las luces por tres segundos antes de iniciar el siguiente ensayo. En este momento, el programa aleatorio volvía a seleccionar una entre las cuatro posibles secuencias. Las sesiones para este grupo también finalizaron después de 100 ensayos o de $30 \mathrm{~min}$.

\section{RESULTADOS}

Los sujetos en el grupo CIS incrementaron el número de reforzadores obtenidos a lo largo del experimento, lo que habla de una mejora en la ejecución de los sujetos. El valor promedio de reforzadores fue 325 en el primer ciclo y 486 en el último ciclo, esto representa un incremento cercano al $50 \%$. Por otro lado, en el grupo SA el número de reforzadores se mantuvo en un promedio de 225 en cada uno de los tres ciclos de 10 sesiones, esto significa que cada secuencia fue seguida por alimento, alrededor de cinco o seis veces en cada sesión.

La figura 1, presenta en dos columnas de gráficas, usando los datos de 
todos los sujetos del grupo CIS, el promedio de la frecuencia observada de cada secuencia en cada uno de los 100 ensayos de la sesión. Una línea vertical divide la gráfica en las dos secciones de 50 ensayos cada una. En todas las gráficas la línea de ajuste representa una media móvil de 5 elementos. La línea gruesa representa el curso de la primera secuencia reforzada, mientras que la línea delgada representa el trayecto de la segunda secuencia reforzada. Los datos de las sesiones que iniciaron con secuencias homogéneas II se presentan en la columna izquierda y las que iniciaron con la secuencia DD en la columna derecha. La forma de las curvas para las secuencias homogéneas y las heterogéneas fue muy diferente. La frecuencia de las secuencias homogéneas fue siempre más alta que la de las heterogéneas.

En la segunda parte de la sesión (ensayos del 51 al 100), es posible apreciar que las curvas se comportan de forma diferente dependiendo de la combinación de secuencias. Por ejemplo, en II-ID, que representa el cambio de la última respuesta de la secuencia, el decremento en la frecuencia de II en la segunda parte, fue rápido y alcanzó niveles cercanos a cero. En el caso II-DI, que supone un cambio en la primera respuesta, el decremento fue rápido; pero se estabilizó en un valor cercano a 10 y se mantuvo por encima de la secuencia ID, que en ese momento producía el reforzador. Para II-DD los cambios fueron rápidos y alcanzaron sus valores máximos alrededor del ensayo 75. Por otro lado, en la columna de la derecha se presentan los resultados de las sesiones que iniciaban con DD, en ellas se aprecian resultados muy similares a los presentados en la columna izquierda. Con la combinación DD-ID (cambio en la primera posición) el nivel de DD se detuvo en un valor cercano a 10 y casi siempre por encima de la secuencia reforzada, mientras que en la combinación DD-DI la frecuencia cayó por debajo del valor cinco. Es difícil decidir, debido al número de sesiones y ensayos, si la diferencia final entre las secuencias heterogéneas favoreció de manera significativa a aquella que recibió el reforzador. Por lo mismo, es cuestionable suponer que las respuestas se integraron como secuencias completas. Lo que sí es claro es que las secuencias homogéneas se adquieren más rápido que las heterogéneas y que este aprendizaje fue más rápido cuando la nueva secuencia difirió de la anterior en la segunda respuesta de la secuencias y no en la primera, como fue reportado por Reid (1994) y Reid et al. (en prensa),

En la figura 2, se presentan los resultados para las sesiones que iniciaron con una secuencia heterogénea, ID en la columna izquierda y DI en la derecha. La diferencia en la forma y valores de la línea de ajuste, entre secuencias homogéneas y heterogéneas también se presentó en este caso. En esta ocasión, durante la primera parte de la sesión, en cuatro de las gráficas la secuencia heterogénea reforzada fue apenas superior a la no reforzada; en el panel DI-II los valores son muy semejantes y en el caso del panel ID-DD, la diferencia favorece a la secuencia no reforzada. Es posible ver que entre las 


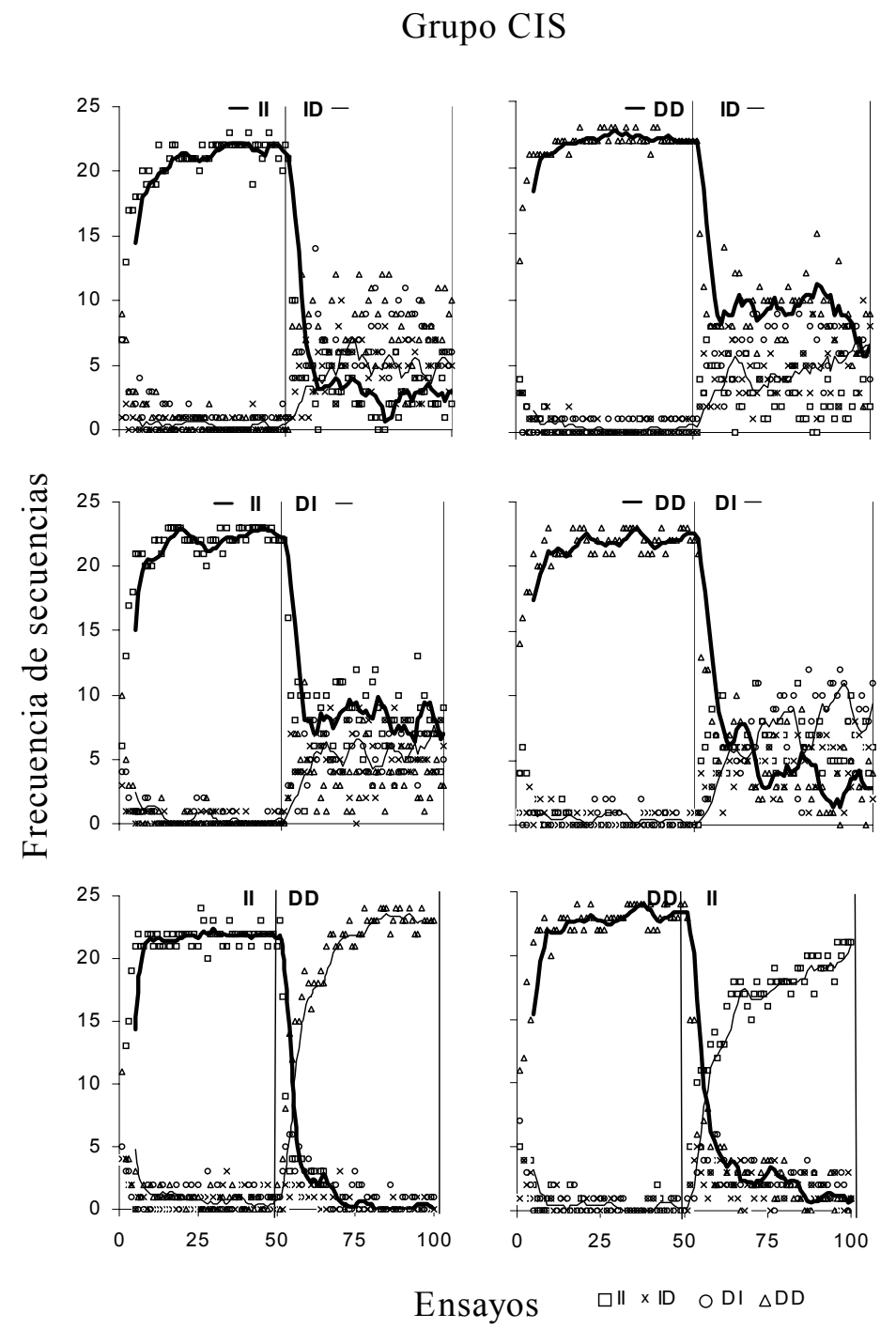

Figura 1. Frecuencia promedio de cada secuencia en cada uno de los 100 ensayos de la sesión. Se utilizaron los datos de los cuatro sujetos del grupo CIS, pero sólo aquellos de las seis sesiones con la combinación de secuencias indicada en cada panel. Una línea vertical señala el ensayo (50) de transición en el reforzamiento de las secuencias. 


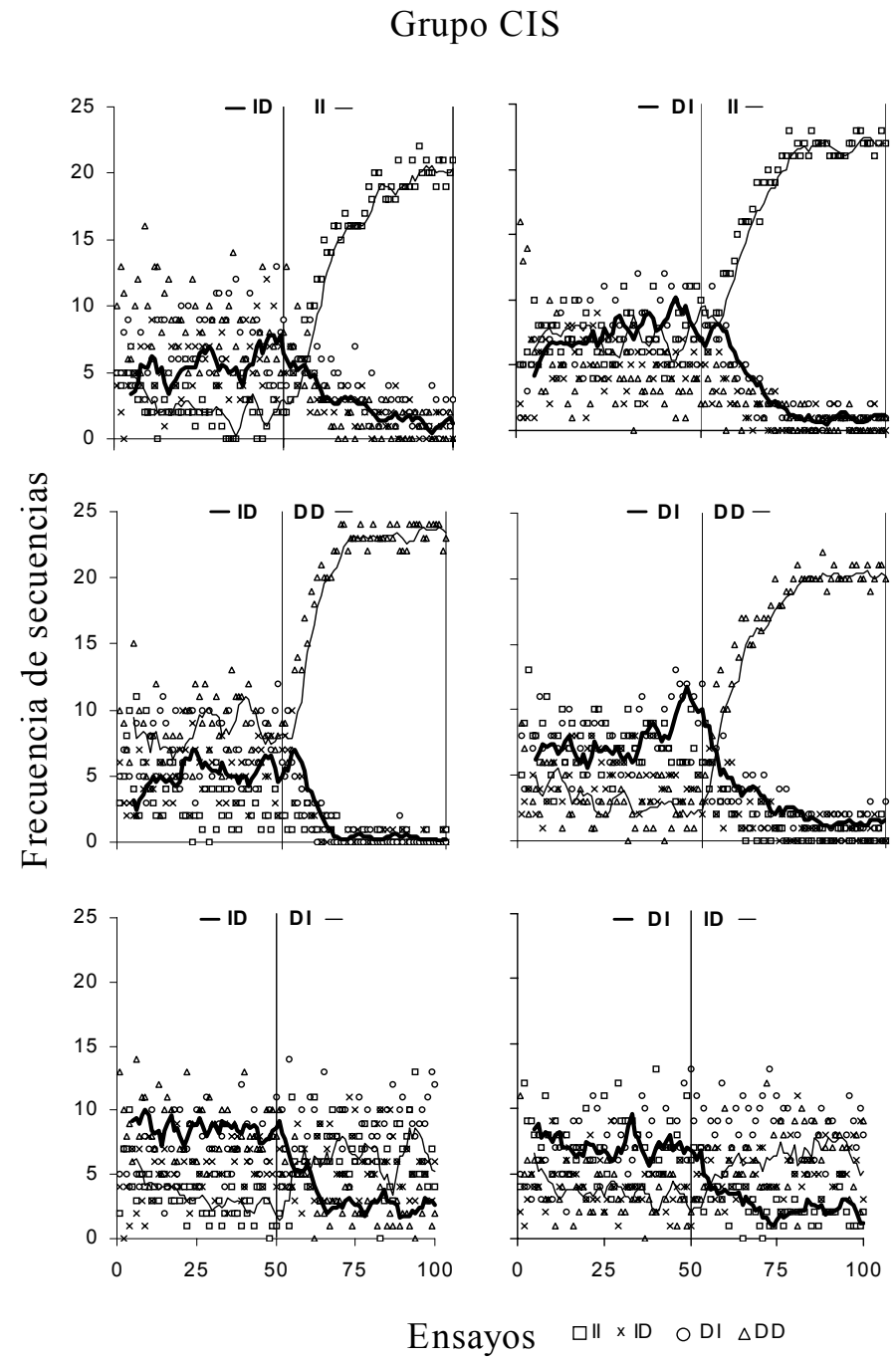

Figura 2. Frecuencia promedio de cada secuencia en cada uno de los 100 ensayos de la sesión. Se utilizaron los datos de los cuatro sujetos del grupo CIS durante las seis sesiones con la combinación de secuencias indicada en cada panel. La línea vertical señala la transición en el reforzamiento de las secuencias. En estas graficas la primera secuencia siempre fue heterogénea. 
gráficas ID-II y ID-DD existen pequeñas diferencias durante la segunda parte de la sesión. Un efecto similar se observa en las gráficas DI-II en la parte superior y de DI-DD en la posición intermedia. En la parte inferior se presentan los resultados para secuencias ID-DI y a la derecha DI-ID. Al comparar estas gráficas con las secuencias homogéneas presentadas en la parte inferior de la figura 1 , se confirma que los sujetos necesitaron más ensayos para adquirir y modificar las secuencias heterogéneas.

En las gráficas de la figura 3, se presenta para el grupo SA, el promedio de la frecuencia de las cuatro secuencias a lo largo de los 100 ensayos, durante el último ciclo de 10 días del experimento. En estas gráficas se aprecia que la distribución de las frecuencias de las cuatro secuencias fue similar entre sí debido, probablemente, a que en este grupo el número de reforzadores fue muy similar para cada secuencia. La diferencia entre secuencias homogéneas y heterogéneas no es notoria en este grupo. El nivel de todas las secuencias fue similar al de las heterogéneas en el grupo CIS, pero es difícil indicar algún orden. Una comparación de estas curvas con aquellas de las figuras 1 y 2 , demuestran que las contingencias de reforzamiento tuvieron un innegable efecto en la distribución de las secuencias.

En la figura 4 se presentan dos gráficas para cada sujeto del grupo CIS. A la izquierda se expone la frecuencia promedio de reforzadores obtenidos por cada secuencia en un ciclo. El número máximo de reforzadores por ciclo fue de 300 y los datos se obtuvieron sumando los reforzadores de todas las sesiones en las que esa secuencia fue seguida por el reforzador y luego obteniendo el promedio para un ciclo. A la derecha, se presenta el promedio de la frecuencia total observada de cada secuencia, aquí el eje se mantuvo en 600 porque ninguna frecuencia fue mayor a esto. En este caso se consideraron todas las sesiones de cada ciclo, lo que implica que se contabilizaron también las sesiones en las que la secuencia no era reforzada. La frecuencia de las secuencias homogéneas (barras obscuras) fue mayor que la de las heterogéneas (barras claras). Esta observación es compatible con las figuras 1 y 2 , esto es, que los sujetos adquirieron más rápido las secuencias homogéneas y con ello obtuvieron más reforzadores que los logrados con las secuencias heterogéneas. En un registro de los datos por ciclo (no presentados aquí) esta diferencia se presentó desde el primer ciclo y aunque varió en cantidad, no lo hizo en la relación que guardan las secuencias entre sí. La distribución de las secuencias en las gráficas del lado derecho tiene una forma semejante a la distribución de los reforzadores obtenidos (izquierda) debido, probablemente, a la restricción impuesta por el programa razón fija uno (RF1), aunque debe recordarse que los sujetos estaban en libertad de ejecutar cualquier secuencia en cada sesión. Para todos los sujetos se aprecia una ligera diferencia entre secuencias homogéneas en favor de DD. También se presentó para tres de los cuatro sujetos, un sesgo en favor de DI en las secuencias heterogéneas. 


\section{Grupo SA}

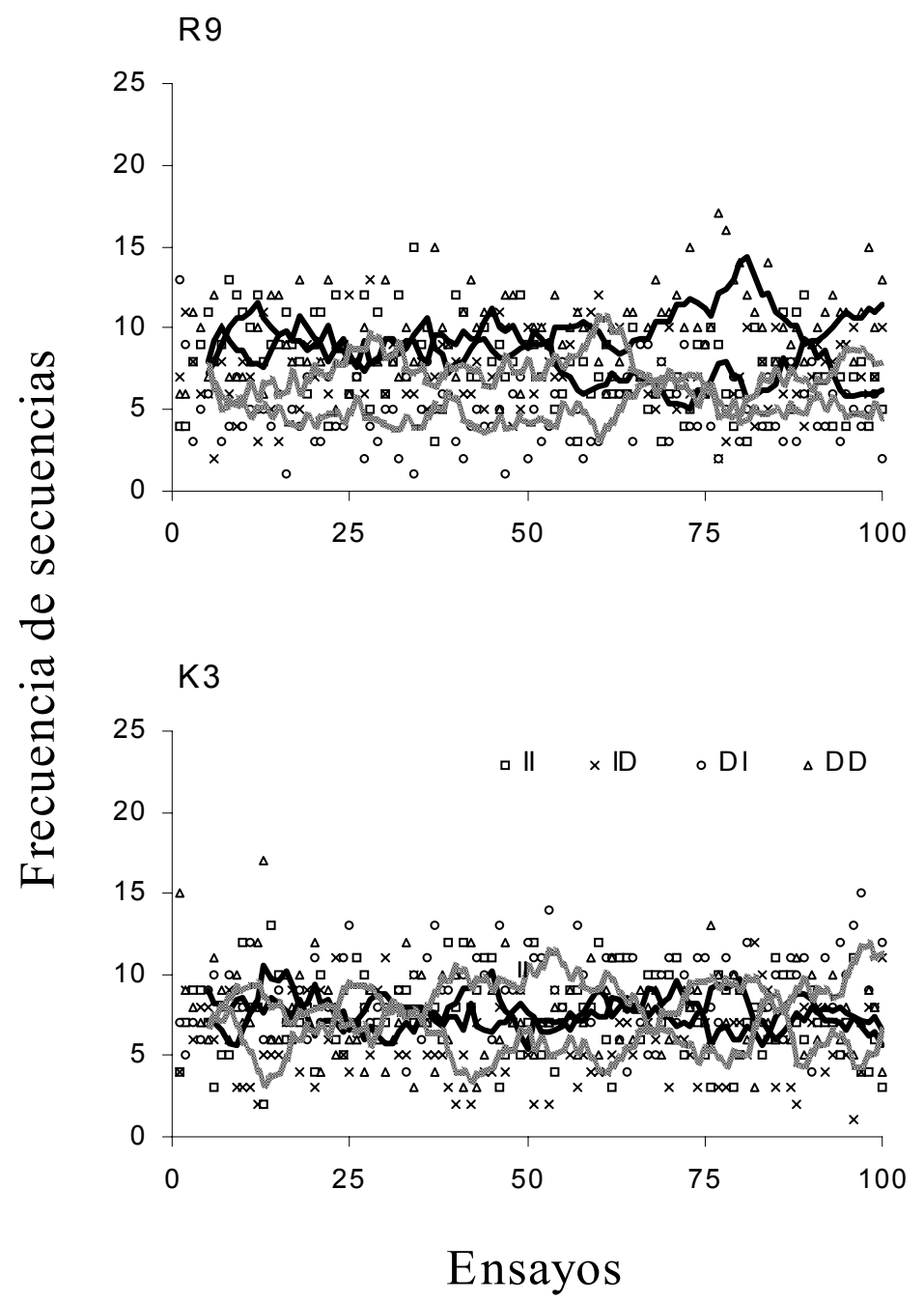

Figura 3. Para el grupo SA se presenta la frecuencia promedio de cada secuencia en cada uno de los 100 ensayos de la sesión. En esta ocasión sólo se promedió la frecuencia de cada secuencia durante las últimas 10 sesiones del grupo. 


\section{Grupo CIS}

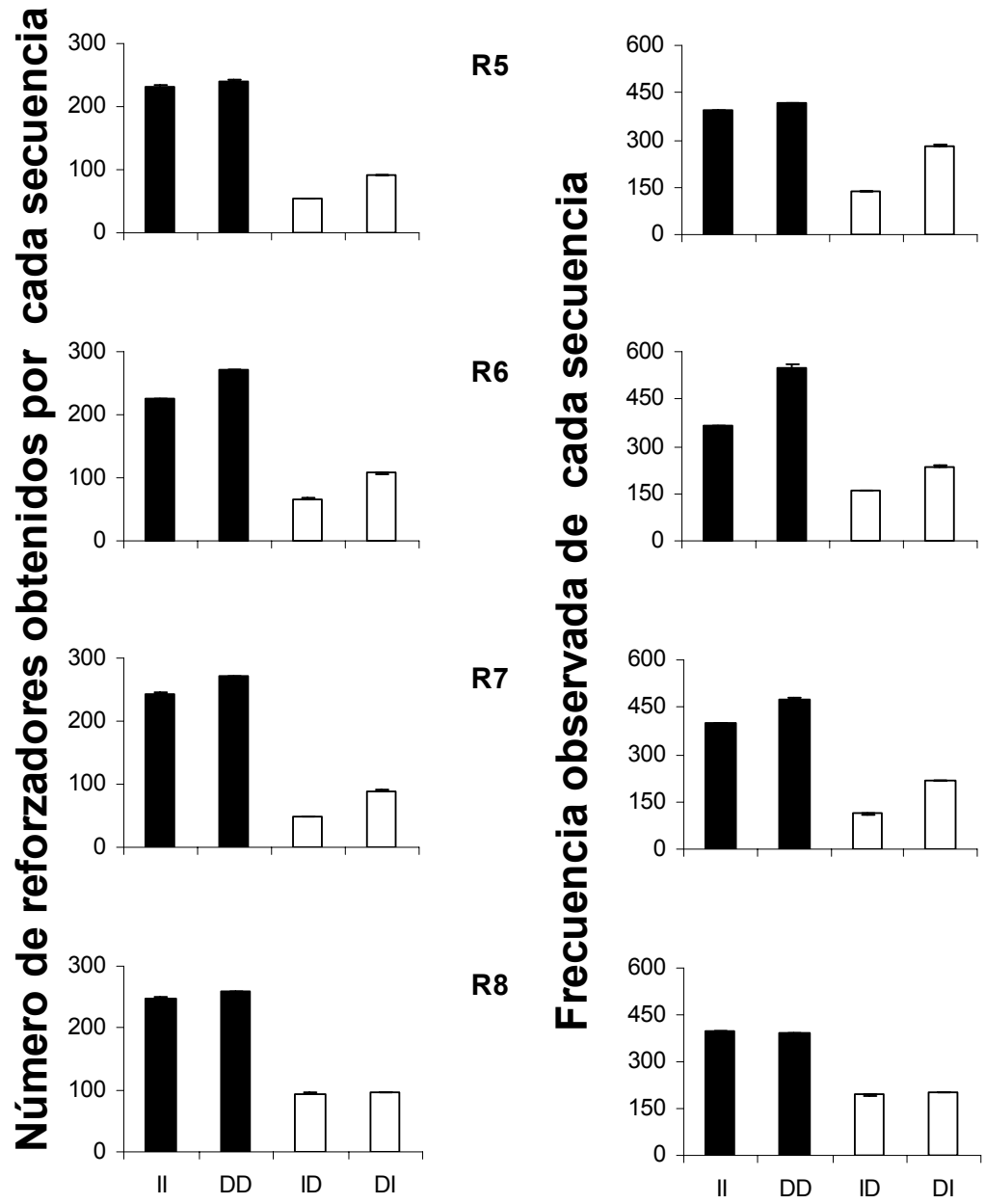

\section{Secuencias}

Figura 4. Frecuencia promedio de reforzadores por ciclo, obtenidos por el grupo CIS con cada secuencia (columna izquierda). Se consideraron sólo las sesiones en las que la secuencia fue reforzada. A la derecha, se presenta el promedio de la frecuencia observada en cada secuencia, considerando todas las sesiones de los seis ciclos. 
Para los dos sujetos del grupo SA, en la figura 5, se hizo el mismo análisis que al grupo CIS. En este grupo, el programa controló el número de reforzadores para cada secuencia y por eso los valores son similares para las cuatro secuencias en las gráficas del lado izquierdo. Las gráficas del lado derecho muestran que la distribución de las cuatro secuencias se presentó también con frecuencias similares, aunque la escala es diferente. La distribución de las frecuencias es diferente a la presentada por los sujetos en el grupo CIS.

En las siguientes dos figuras se muestra el promedio de la frecuencia de las secuencias, utilizando los datos de todos los ciclos. En ellas se especifica si la secuencia reforzada era homogénea (II+, DD+) o heterogénea (ID+, DI+). En la figura 6 se presentan los resultados para las sesiones en las que el reforzador correspondió a una secuencia homogénea (representada con una barra oscura). Para cada sujeto se presentan cuatro gráficas. En la primera columna (II-X) se exponen los resultados de sesiones en las que se reforzó II durante los primeros 50 ensayos, sin importar cuál fue reforzada durante la segunda parte de la sesión. La segunda columna (X-II) representa los datos cuando se reforzó II durante la segunda mitad de la sesión independientemente de la secuencia reforzada en la primera parte. De forma similar, en las dos siguientes columnas se reportan los resultados de las sesiones DD-X y X-DD. Esta figura muestra patrones similares para los cuatro sujetos y no parece haber grandes diferencias entre ellas. Sin embargo, las frecuencias de las secuencias reforzadas en la primera parte son ligera pero consistentemente mayores que las frecuencias de secuencias reforzadas en la segunda parte de la sesión.

La figura 7 muestra la misma relación presentada en la figura anterior pero con los resultados de aquellas sesiones en las que una secuencia heterogénea era reforzada. En la primera columna se presentan para todos los sujetos, los resultados de las sesiones con reforzamiento para la secuencia ID en la primera parte (ID-X). En la segunda columna se muestran las sesiones con la combinación X-ID en las que se reforzaba ID en los últimos 50 ensayos de la sesión. En las dos columnas de la derecha se hace lo mismo con los datos para las sesiones con DI-X y X-DI. La distribución es diferente a la observada en la figura 6 . La diferencia entre la frecuencia de la secuencia reforzada y las otras, no es tan clara como en las gráficas previas. Ahora, se observan pequeñas diferencias entre las barras que representan secuencias heterogéneas $y$, en muchos casos existe un sesgo en favor de DI, incluso cuando la condición era de reforzamiento para ID. Asimismo, se presentó el efecto debido a la proximidad de la segunda respuesta de la secuencia heterogénea con el reforzador, esto es, si el reforzador dependía de la secuencia ID, entonces la barra correspondiente a DD (barra con líneas diagonales) es siempre mayor que la barra para II. Cuando el reforzador estaba asociado a la secuencia DI, la diferencia favorece de manera consistente a la barra que representa a II. 


\section{Grupo SA}
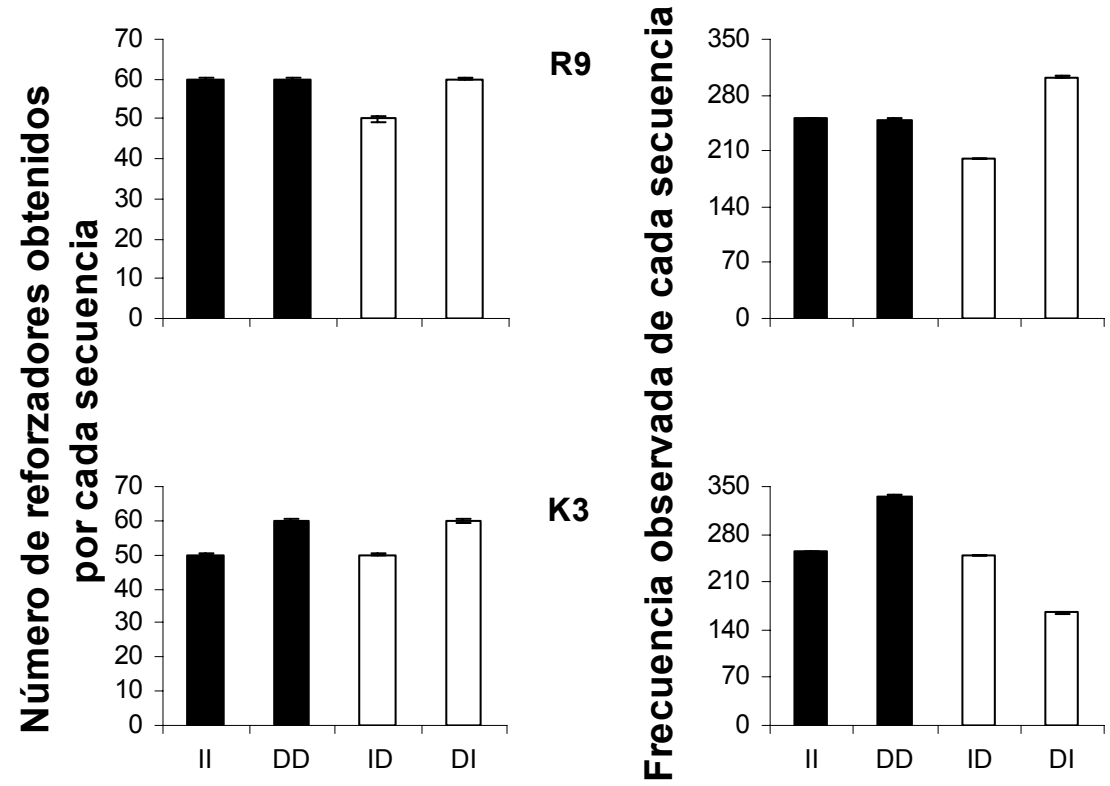

\section{Secuencias}

Figura 5. Frecuencia promedio de reforzadores que siguieron a cada secuencia en el grupo SA. Para este grupo, los tres bloques fueron de 10 sesiones cada uno. A la derecha se presenta la frecuencia promedio de cada secuencia, considerando todos los bloques. 


\section{Secuencias Homogéneas}

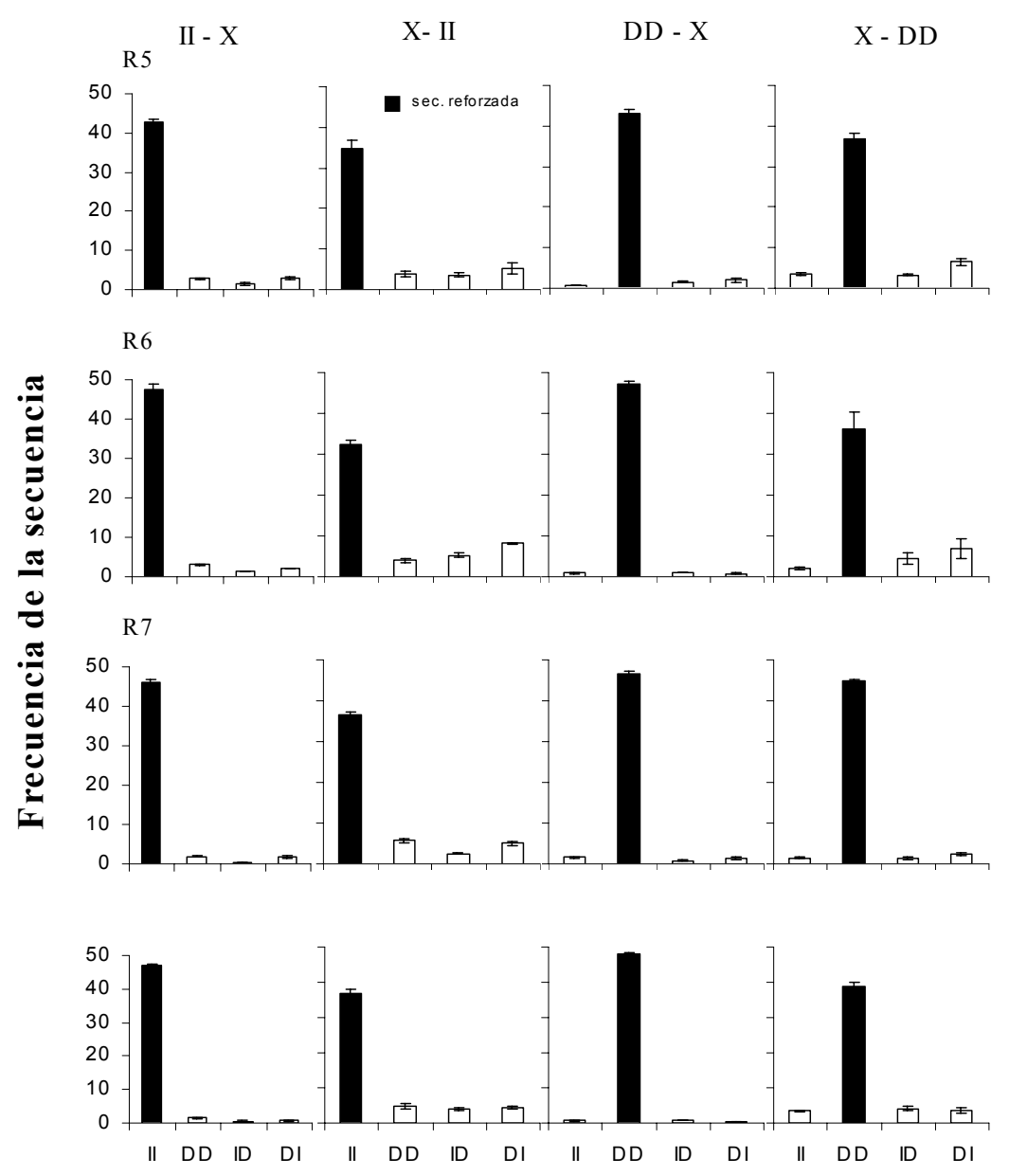

\section{Secuencias}

Figura 6. Frecuencia promedio de cada secuencia (Grupo CIS), cuando se reforzaba una de las dos homogéneas II o DD. Las columna separan secuencias reforzadas en los primeros 50 ensayos (II-X) de aquellas reforzadas durante los últimos ensayos (X-DD). La barra oscura representa la secuencia reforzada y la barra con líneas diagonales es la secuencia homogénea asociada con el reforzador. 


\section{Secuencias Heterogéneas}
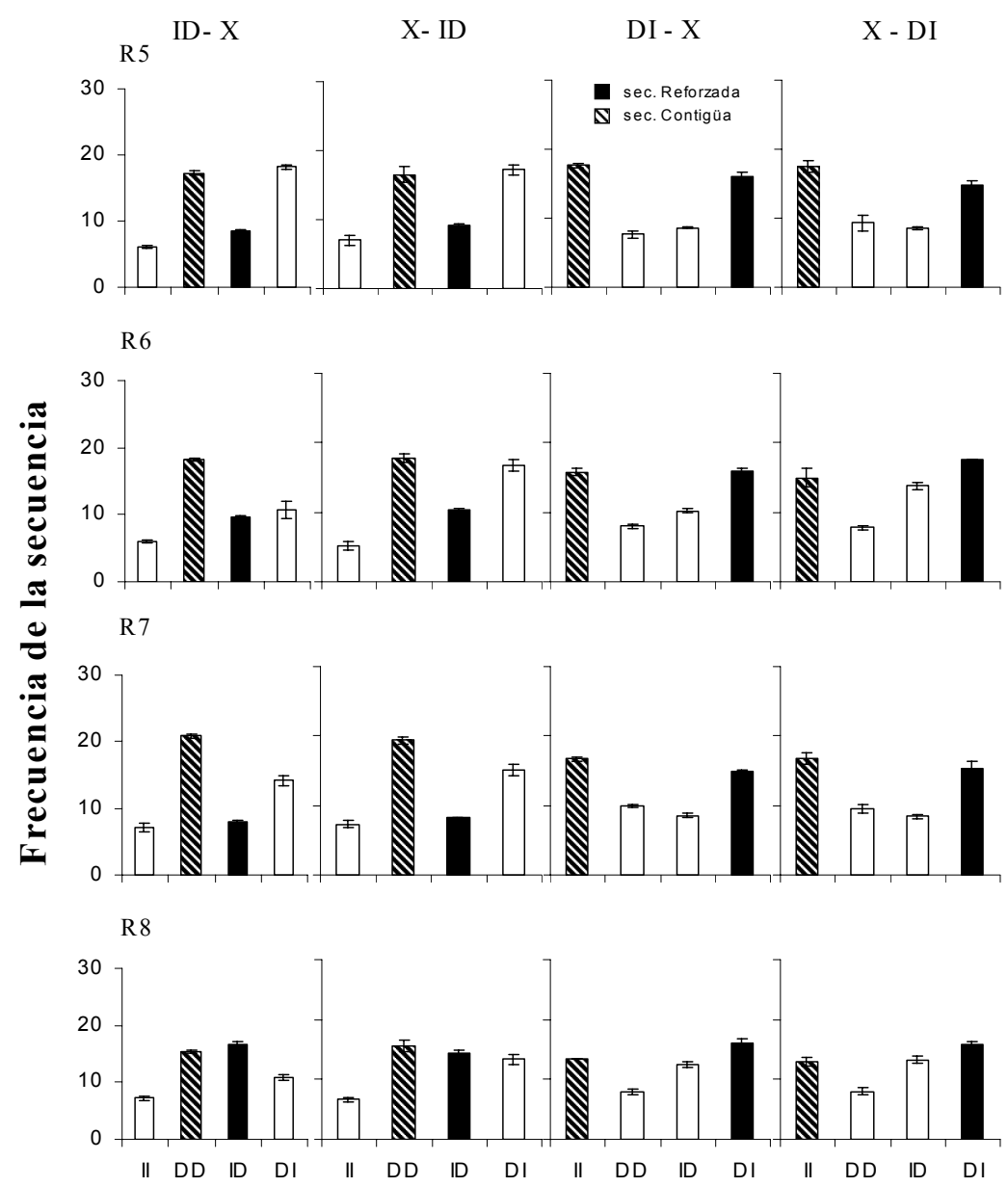

Secuencias

Figura 7. Frecuencia promedio de cada secuencia (Grupo CIS), al reforzar una de las heterogéneas ID o DI. Las columnas presentan por separado las secuencias reforzadas durante los primeros 50 ensayos (ID-X) de las reforzadas durante los últimos ensayos $(X-D I)$. La barra en negro representa la secuencia reforzada y la barra con líneas diagonales es la secuencia homogénea asociada con el reforzador. 
En el grupo CIS, el diseño controló el número de oportunidades para que el reforzador siguiera a cada secuencia, sin embargo esto no determinó directamente el número de pellas obtenidas con cada secuencia. Por otro lado, en el grupo SA se controló el número de reforzadores para cada secuencia. Ambos arreglos, tenían la intención de controlar el que cada palanca fuera seguida de un número similar de reforzadores. Para confirmarlo, en la tabla 2 se muestra, para cada sujeto, el número de reforzadores por ciclo que siguió a las respuestas en la palanca I y en la D. Las columnas especifican también si la respuesta fue ejecutada en la primera o en la segunda posición de la secuencia. Todos los valores que se utilizaron para obtener los promedios fueron sometidos a una prueba t para saber si existía una diferencia significativa entre ellos. Los resultados de la prueba se presentan para cada sujeto, en el lado derecho de la tabla. En la parte superior, se presentan los resultados para las ratas del grupo CIS, los valores asociados con una $p<$ .05 , se identifican con un asterisco, si la $p<.01$ con dos asteriscos y además se indica con una letra a que palanca favoreció. Como se aprecia, existió una diferencia en tres casos a favor de la palanca $D$ en la primera posición. Las condiciones en este grupo generaron un ligero sesgo en favor de las secuencias que incluyen la respuesta $\mathrm{D}$ en su primera posición DD y DI. En cambio, en el caso del grupo SA (parte inferior derecha de la tabla 2) utilizando la misma prueba, ninguna de las diferencias alcanzó niveles estadísticamente significativos.

Para analizar el estado estable alcanzado por los sujetos, se utilizaron los datos del último ciclo de sesiones de cada grupo, 12 sesiones para el grupo CIS y 10 sesiones para el SA. La tabla 3a, presenta el promedio de reforzadores que siguieron a la operación de la palanca I y la $\mathrm{D}$, tanto en la primera como en la segunda posición. Para esta tabla se utilizaron los datos del último ciclo del experimento, considerando que representan el momento de mayor estabilidad. La tabla demuestra que en ambos grupos la cantidad de reforzadores para cada palanca fue muy semejante; sin embargo se observa que la distribución de las secuencias fue muy diferente entre los grupos. Para complementar este análisis se calculó la frecuencia con la que se presentó cada una de las secuencias así como el número de reforzadores ganados con cada una de ellas. Estas frecuencias se presentan en la tabla 3.b, para este cálculo se utilizaron los datos del experimento completo, es decir los seis ciclos de 12 sesiones para el grupo CIS y de tres ciclos de 10 sesiones para el grupo SA. La última columna para el grupo CIS muestra la frecuencia de cada secuencia, en aquellas sesiones en las que no eran seguidas por el reforzador. Los resultados generales indican una diferencia entre grupos que puede ser atribuida a las condiciones de entrega del reforzador. En el grupo CIS las diferencias entre las secuencias parecen ser el resultado de su estructura diferente. 
Tabla 2. Promedio de reforzadores por sesión que siguió a cada palanca en cada posición de la secuencia. Se presentan los datos para cada sujeto de ambos grupos por ciclo. Los ciclos para el grupo CIS consistieron de de 12 sesiones y para el grupo SA, de 10 sesiones. Del lado derecho de la tabla se exponen los resultados de una prueba $t$ aplicada a los datos, indicando con negritas las diferencias con un valor de $p<.05$ y se indica dentro de un paréntesis, la palanca a la que favoreció la diferencia.

\begin{tabular}{|c|c|c|c|c|}
\hline \multirow{4}{*}{ Sujetos } & \multicolumn{4}{|c|}{ Reforzadores que siguieron a cada palanca } \\
\hline & \multicolumn{4}{|c|}{ Grupo CIS } \\
\hline & \multicolumn{2}{|c|}{$1^{\circ}$ posición } & \multicolumn{2}{|c|}{$2^{\circ}$ posición } \\
\hline & I & D & I & D \\
\hline \multirow{5}{*}{$\mathrm{R} 5$} & 37 & 39 & 41 & 35 \\
\hline & 42 & 52 & 50 & 44 \\
\hline & 45 & 62 & 54 & 53 \\
\hline & 57 & 60 & 64 & 53 \\
\hline & 54 & 57 & 57 & 53 \\
\hline \multirow{7}{*}{ R6 } & 52 & 63 & 58 & 57 \\
\hline & 30 & 56 & 35 & 50 \\
\hline & 48 & 65 & 53 & 60 \\
\hline & 49 & 65 & 58 & 56 \\
\hline & 58 & 61 & 57 & 61 \\
\hline & 57 & 62 & 61 & 58 \\
\hline & 50 & 70 & 67 & 53 \\
\hline \multirow{5}{*}{ R7 } & 29 & 48 & 34 & 42 \\
\hline & 54 & 58 & 58 & 53 \\
\hline & 48 & 63 & 59 & 52 \\
\hline & 54 & 61 & 59 & 56 \\
\hline & 53 & 65 & 63 & 56 \\
\hline \multirow{7}{*}{$\mathrm{R} 8$} & 56 & 65 & 61 & 60 \\
\hline & 40 & 47 & 44 & 43 \\
\hline & 54 & 62 & 62 & 54 \\
\hline & 51 & 63 & 56 & 59 \\
\hline & 60 & 62 & 59 & 63 \\
\hline & 72 & 56 & 59 & 69 \\
\hline & 65 & 64 & 65 & 64 \\
\hline
\end{tabular}

\begin{tabular}{ccc}
\hline \multicolumn{3}{c}{ Valor obtenido en las pruebas $\boldsymbol{t}$} \\
\hline Sujetos & \multicolumn{2}{c}{ Grupo CIS } \\
\cline { 2 - 3 } & $\mathbf{1}^{\circ}$ posición & $\mathbf{2}^{\circ}$ posición \\
\hline R5 & $\mathbf{1 . 3 8}^{*} \mathbf{D}$ & 0.874 \\
R6 & $\mathbf{2 . 7 2}^{*}$ D & 0.193 \\
R7 & $\mathbf{1 . 9 1}^{* *} \mathbf{D}$ & 0.407 \\
R8 & 0.381 & 0.235 \\
\hline
\end{tabular}

\begin{tabular}{ccccc} 
& \multicolumn{4}{c}{ Reforzadores que siguieron a cada palanca } \\
\cline { 2 - 5 } & \multicolumn{4}{c}{ Grupo SA } \\
\hline Sujetos & $\mathbf{1}^{\circ}$ posición & \multicolumn{2}{c}{$\mathbf{2}^{\circ}$ posición } \\
\hline \multirow{3}{*}{ R9 } & I & D & I & D \\
\hline \multirow{4}{*}{ K3 } & 13 & 12 & 12 & 13 \\
& 12 & 11 & 13 & 10 \\
& 12 & 9 & 9 & 13 \\
\cline { 2 - 5 } & 10 & 11 & 10 & 11 \\
& 11 & 12 & 11 & 11 \\
& 11 & 14 & 12 & 12 \\
\hline
\end{tabular}

\begin{tabular}{ccc}
\hline \multicolumn{3}{c}{ Valor obtenido en las pruebas $t$} \\
\hline \multirow{2}{*}{ Sujetos } & \multicolumn{2}{c}{ Grupo SA } \\
\cline { 2 - 3 } & $\mathbf{1}^{\circ}$ posición & $\mathbf{2}^{\circ}$ posición \\
R9 & 0.132 & 0.531 \\
K3 & 0.140 & 0.917 \\
\hline
\end{tabular}

Nota: ${ }^{*} p<.05^{* *} p<.01$ 
Tabla 3. Para ambos grupos, el panel 3.a, muestra el promedio de reforzadores durante el último ciclo, que siguieron a las respuestas en cada palanca, considerando su posición en la secuencia. En el panel 3.b se presenta el promedio de reforzadores obtenidos por cada secuencia junto con el promedio de cada secuencia en un ciclo. Para este cálculo se utilizaron los datos de todas las sesiones del experimento.

\begin{tabular}{|c|c|c|c|c|c|c|}
\hline & & \multicolumn{2}{|c|}{ Grupo CIS } & & & \\
\hline & \multirow{4}{*}{$\begin{array}{l}\text { Palanca I } \\
\text { Palanca D }\end{array}$} & $1^{\circ}$ posición & $2^{\circ}$ posición & & & \\
\hline & & 303 & 333 & & & \\
\hline & & 356 & 326 & & & \\
\hline & & \multicolumn{2}{|c|}{ Grupo SA } & & & \\
\hline & Palanca I & 114 & 116 & & & \\
\hline & Palanca D & 103 & 125 & & & \\
\hline \multicolumn{7}{|l|}{ Tabla $3 . b$} \\
\hline & \multicolumn{2}{|c|}{ Grupo SA } & & \multicolumn{3}{|c|}{ Grupo CIS } \\
\hline Secuencia & $\begin{array}{c}\text { Frecuencia } \\
\text { Total }\end{array}$ & $\begin{array}{c}\text { Reforzadores } \\
\text { obtenidos }\end{array}$ & & $\begin{array}{c}\text { Frecuencia } \\
\text { Total }\end{array}$ & $\begin{array}{c}\text { Reforzadores } \\
\text { obtenidos }\end{array}$ & $\begin{array}{c}\text { Secuencias } \\
\text { No reforzadas }\end{array}$ \\
\hline II & 252 & 57 & & 388 & 237 & 151 \\
\hline DD & 225 & 58 & & 150 & 66 & 84 \\
\hline ID & 234 & 56 & & 227 & 96 & 131 \\
\hline DI & 293 & 58 & & 435 & 260 & 175 \\
\hline
\end{tabular}

\section{DISCUSIÓN}

El trabajo logró su propósito de controlar las oportunidades de reforzamiento para un grupo y el número de reforzadores para otro grupo. Esto permitió analizar en condiciones comparables, los procesos involucrado en la adquisición y adaptación a condiciones que variaron en cada sesión, mostrando además el peso que tiene la estructura de las secuencias.

Los resultados muestran patrones que revelan una diferencia entre los grupos. El grupo CIS desarrolló una diferencia muy clara entre las frecuencias de secuencias homogéneas y las heterogéneas, a favor de las primeras. En cambio en el grupo SA la restricción de obtener un número similar de reforzadores con cada secuencia provocó que las frecuencias fueran similares. Lo anterior podría estar reflejando el uso de diferentes estrategias. Mientras que en el grupo CIS la discriminación de la palanca en la cual repetir las respuestas (secuencias homogéneas) se adquirió y modificó muy rápido, con las secuencias heterogéneas la adquisición de una alternación exitosa que 
demostrara una discriminación entre ellas se desarrolló muy lentamente. En el caso del grupo SA, las respuestas parecieron seguir más un esquema aleatorio que sin embargo permitió obtener los reforzadores programados.

Se confirmó la presencia de un efecto debido a la cercanía de la última respuesta con el reforzador (ver figura 7) reportado con anterioridad por Grayson y Wasserman (1979) en un trabajo con palomas usando también secuencias de dos respuestas, que intentaba demostrar la generalidad de los principios operantes de respuestas discretas a grupos de respuestas. En el trabajo de Grayson y Wasserman, el efecto es explicado como resultado del reforzamiento prolongado de una misma secuencia; lo que resulta en una probabilidad de respuestas diferente y siempre a favor de la más cercana al reforzador. En el presente trabajo se observó el mismo efecto, pero en condiciones que implicaban la ejecución de secuencias diferentes en cada sesión. Es por esto que se considera que la frecuencia observada de la secuencia homogénea asociada al reforzador es el resultado de un efecto al nivel de las respuestas que conforman la secuencia y no necesariamente el resultado de la formación de una unidad mayor debida a un entrenamiento prolongado. Además, fue claro que los nuevos patrones de conducta dependen no sólo de ser reforzados sino, también, de la estructura de la secuencia previa y que la frecuencia de la secuencia sometida a la condición de extinción varió dependiendo de la estructura de la nueva secuencia que se tenía que aprender; lo cual confirma lo propuesto por Reid (1994) y Reid et al. (en prensa)

El actual trabajo permite detectar la presencia de procesos a nivel de las respuestas en la formación de pautas conductuales, confirmando por ejemplo la propuesta de Catania (1971) en el sentido de que el efecto del reforzador actúa no sólo en la respuesta inmediata previa a él sino en todas aquellas que la anteceden. El reforzador actúa sobre cada elemento de la secuencia y cada respuesta contribuye de forma independiente a la futura tasa total de la secuencia. La contribución de cada respuesta es inversa a su distancia al reforzador. La descripción de los resultados y de los procesos en este trabajo, se hizo a partir del número de reforzadores sin implicar necesariamente el concepto de fuerza, para evitar el argumento de que la fuerza o la frecuencia dependen exclusivamente del número de reforzadores. En el grupo SA por ejemplo, se presentaron un número similar de reforzadores después de la palanca izquierda o derecha y después de cada secuencia, en consecuencia se observaron frecuencias similares entre secuencias homogéneas y heterogéneas. Lo anterior sugiere una relación del tipo I $=D$, entonces II $=D D$ e incluso ID = DI. Sin embargo, en el grupo CIS que brindaba un número igual de oportunidades (pero no necesariamente de reforzadores) se muestra que a pesar de la semejanza en el número de reforzadores que siguieron a cada palanca individual, en el caso de las secuencias, este número fue distinto para secuencias homogéneas y heterogéneas. Los resultados muestran una 
mayor frecuencia de reforzadores obtenidos con secuencias homogéneas vs heterogéneas. Incluso se observaron diferencias entre el número de reforzadores logrados con secuencias del mismo tipo, por ejemplo (II < DD) y (ID < DI). Estas diferencias coinciden con la frecuencia total observada (tabla 3.b) de cada secuencia. Es necesario, en este punto, considerar la restricción que impone el programa (RF1) usado para entregar el reforzador. Al existir una relación uno a uno entre la exigencia del programa y el número de reforzadores, el programa puede ser la causa de la distribución de la frecuencia total. Sin embargo, debe considerarse el hecho de que la distribución de las secuencias no reforzadas mantuvo la misma proporción que la de los reforzadores obtenidos (ver última columna tabla 3.b). Este último dato, apoya la evidencia existente (Fetterman y Stubb, 1982) de que la frecuencia de reforzadores ejerce control sobre la frecuencia de las secuencias cuando se utilizan programas diferentes.

En resumen, para el grupo CIS el aprendizaje y la ejecución de secuencias homogéneas (aquellas que requieren de la discriminación del lugar y repetir la respuesta hasta obtener el reforzador) fue rápida y estable. Por otro lado, la ejecución de las secuencias heterogéneas, que involucra la discriminación del orden en el que hay que ejecutar las respuestas para obtener el reforzador, fue errática y sugiere que no se logró tal discriminación. De hecho, el sesgo observado en la figura 7 a favor de la secuencia DI, y que no desapareció aun cuando el reforzador dependía de ID, induce a pensar que estas secuencias nunca se formaron como secuencias integradas. Por tanto, se puede afirmar que los procesos actuaron a nivel de las respuestas y que ni las secuencias heterogéneas en el grupo CIS ni ninguna de las secuencias en el grupo SA, se comportaron como secuencia integradas en nuevas unidades.

Finalmente se considera que el estudio de las condiciones en las cuales se adquieren, mantienen y modifican las secuencias conductuales, representa un reto natural al grado de generalización de nuestro conocimiento sobre la forma en la que las consecuencias afectan la conducta de los organismos (Reid, 2003). La propuesta del presente estudio facilitó evaluar, en circunstancias comparables, algunos de los procesos que trabajan en la formación de patrones simples, en un medio con cambios frecuentes. Estas condiciones parecen favorecer la acción de procesos al nivel cada respuesta, aunque no se descarta que en condiciones estables, con cambios de contingencia poco frecuentes se puedan observar procesos que involucren a la secuencia completa (Pisacreta, 1982; Reed, 1994; Schwartz, 1980, 1981; Terrace, 1991). De hecho, existen pruebas de que ambos procesos trabajan en un orden e incluso de manera simultánea (Bachá, Reid y Mendoza, en prensa) y por lo tanto se prevé que un modelo del mecanismo responsable de la formación de patrones conductuales a partir de respuestas discretas deberá ser capaz de integrar ambos tipos de procesos. 


\section{REFERENCIAS}

Bachá G. Reid, A. K. y Mendoza (en prensa). Resurgence of Complex Behavioral Units. Journal of the Experimental Analysis of Behavior

Boren, J. J., \& Devine, D. D. (1968). The repeated acquisition of behavioral chains. Journal of the Experimental Analysis of Behavior, 2(6), 651-660.

Catania, A. C. (1971). Reinforcement schedules: The role of responses preceding the one that produces the reinforcer. Journal of the Experimental Analysis of Behavior, 15(3), 271-287.

Catania, A. C. (1998). Learning (Fourth ed.). Upper Saddle River, N.J.: Prentice Hall.

Fetterman, J. G., \& Stubbs, D. A. (1982). Matching, maximizing, and the behavioral unit: Concurrent reinforcement of response sequences. Journal of the Experimental Analysis of Behavior, 37(1), 97-114.

Grayson, R. J., \& Wasserman, E. A. (1979). Conditioning of two-response patterns of key pecking in pigeons. Journal of the Experimental Analysis of Behavior, 31(1), 23-29.

Kelleher, R. T. (1966). Chaining and conditioned reinforcement. In W. K. Honig (Ed.), Operant Behavior: Areas of Research and Application. Englewood Cliffs: Prentice Hall.

Pisacreta, R. (1982). Some factors that influence the acquisition of complex, stereotyped response sequences in pigeons. Journal of Experimental Analysis of Behavior. 37,359-369

Polidora, V. J. A. (1963). A sequential response method of studying complex behavior in animals and its application to the measurement of drug effects. Journal of Experimental Analysis of Behavior, 6(2), 271-277.

Reed, P. (1994). Influence of the cost of responding on human causality judgments Memory \& Cognition, 22(2), 243-248.

Reid, A. K. (1994). Learning new response sequences. Behavioural Processes, 32, 147-162.

Reid, A. K. (2003). Understanding Response Sequences. Paper presented the SQAB.

Reid, A. K., Chadwick C. Z., Dunham, M. \& Miller A. (2001). The development of functional response units: The role of demarcating stimuli. Journal of the Experimental Analysis of Behavior, 76(3), 303-320.

Reid, A. K., Dixon, R. A., \& Gray, S. (in press). Variation and selection in response structures. In N. Innis (Ed.). Reflections on Adaptive Behavior: Essays in Honor of J. E. R. Staddon. Cambridge, MA: MIT Press.

Schneider, S. M., \& Morris, E. K. (1992). Sequences of spaced response: Behavior units and the role of contiguity. Journal of the Experimental Analysis of Behavior, 58(3), 537-555.

Schwartz, B. (1980). Development of complex, stereotyped behavior in pigeons. Journal of the Experimental Analysis of Behavior, 33, 153-166.

Schwartz, B. (1981). Reinforcement creates behavioral units. Behaviour Analysis Letters, 1, 33-41.

Shimp, C. P. (1979). The local organitation of behavior: method and theory. In M. D. 
Zeiler \& P. Harzem (Eds.), Reinforcement and the Organitation of Behavior (pp. 261-298). New York: Willey.

Skinner, B. F. (1938). The Behavior of Organisms. New York: Appleton Century Crofts.

Terrace, H. S. (1991). Chunking during serial learning by a pigeon: I. Basic evidence. Journal of Experimental Psychology: Animal Behavior Processes., 17, 81-93.

Thompson, D. M. (1973). Repeated acquisition as a behavioral base line for studying drug effects. The Journal of Pharmacology and Experimental Therapeutics, 184(2).

Thompson, D. M. (1975). Repeated acquisition of response sequences: Stimulus control and drugs. Journal of the Experimental Analysis of Behavior, 23(3), 429-436.

Wasserman, E. A., Deich, J. D., \& Cox, K. E. (1984). The learning and memory of response sequences. In M. I. Commons, R. J. Herrnstein \& A. R. Wagner (Eds.) Quantitative Analysis of Behavior. Vol. IV: Discrimination Processes. (pp. 99-113). Cambridge, MA: Ballinger. 